\title{
Development of an in-solution observation method using atmospheric scanning electron microscopy (ASEM)
}

\author{
— Interdisciplinary research between semiconductor fabrication technology \\ and biological electron microscopy -
}

\author{
Toshihiko OGURA ${ }^{1}$, Hidetoshi NISHIYAMA ${ }^{2}$, Mitsuo SUGA ${ }^{2}$ and Chikara SATO ${ }^{3 *}$
}

[Translation from Synthesiology, Vol.8, No.3, p.116-126 (2015)]

\begin{abstract}
Protein complexes in cells and tissues play critical roles in various physiological functions, including embryogenesis and signal processing. To observe the dynamics of protein complexes, high-resolution and high-throughput electron microscopy (EM) in aqueous solution is required. However, standard EM requires the sample to be in a vacuum. With ASEM, an inverted scanning electron microscope (SEM) observes the wet sample from beneath an open dish while an optical microscope (OM) observes it from above. The disposable dish with a silicon nitride film window can hold a few milliliters of culture medium, allowing various types of cells to be cultured in a stable environment. This system was used to develop in situ correlative OM/SEM immuno-microscopy in liquid. Using this method, we have observed a dynamic string-like gathering of STIM1 on the endoplasmic reticulum in Jurkat T cells in response to Ca ${ }^{2+}$ store depletion. We have also observed filamentous-actin (F-actin) and tubulin in the growth cones of primary-culture neurons, as well as in synapses. Further, we have monitored in-situ electrochemical reactions in electrolytes, and the melting and solidification of solder using ASEM.
\end{abstract}

Keywords : Correlative Light and Electron Microscopy (CLEM), microtubule, STIM1, immuno-electron microscopy, electrochemistry

\section{Introduction}

Many physical phenomena and chemical reactions occur in an aqueous environment, and our most distant ancestor was born in the sea. Therefore, to observe events in an aqueous environment at high resolution is important for both physics and biological research. For example, proteins are essential factors that support the biological function of our bodies, and many move dynamically inside cells. TRPV2 ion channels are normally integrated in the intracellular membrane, and when they sense a stimulus, they move to the cell surface and start to function within a few seconds. ${ }^{[1]}$ In recent years, such quick acting proteins have been found in large numbers, and they perform physiological functions by associating with and dissociating from other proteins. Further, many protein complexes associate directly or indirectly with the cytoskeleton and are specifically localized in cells. Quick sample preparation and high-resolution observation are required to clarify the behavior and function of complexes with multiple components. So-called high-throughput EM observation would be such a method. However, in conventional electron microscopy (EM), complicated pretreatment procedures are necessary to prepare the sample for observation in the vacuum of an electron microscope.

The atmospheric scanning electron microscope (ASEM) that we developed is capable of observing cells labeled with heavy metal or fluorescence directly in water without pretreatment (Fig. 1). This allows high-throughput and high-resolution observation. It is also excellent for immuno-EM where the target molecule is labeled with gold-labeled antibodies, because antibody antigenicity is conserved in under aqueous conditions, and because the natural structure in hydrophilic conditions can be observed; hydrophobic treatment is not required. Moreover, ASEM can be used for correlative light and electron microscopy (CLEM), as it allows correlative observation of the sample by an optical microscope (OM) and by EM. The speed and high resolution with which it is possible to directly observe samples immersed in solution, are expected to enable various medical diagnoses. Although ASEM was developed for bio-research, the demands for observation in gas and liquid environments are high in the physical sciences and nanotechnology, and its applications are expanding widely.

\section{Outline of conventional EM for gas and liquid and the ASEM system}

\subsection{Conventional EM and its limits}

Epon thin-sectioning transmission electron microscopy (TEM) is used to observe intracellular structures and cellular organelles and molecular complexes, providing much higher resolution than conventional OM. This comparatively high resolution has allowed the discovery of various biologically

1. Biomedical Research Institute, AIST Tsukuba Central 2, 1-1-1 Umezono, Tsukuba 305-8568, Japan, 2. Advanced Technology Division, JEOL Ltd. 3-1-2 Musashino, Akishima 196-8558, Japan, 3. Biomedical Research Institute, AIST Tsukuba Central 6 and 2, 1-1-1 Higashi, Tsukuba 305-8566, Japan * E-mail: ti-sato@aist.go.jp

Original manuscript received August 5, 2013, Revisions received January 9, 2015, Accepted January 27, 2015 
and medically important mechanisms and phenomena. However, it is necessary to maintain the microscope column under vacuum to prevent the electron beam from being scattered. Therefore, the samples are normally subjected to pretreatments that may require several hours to several days to ensure durability in a vacuum. Since the treatments often involve dehydration and resin embedding, they could affect delicate hydrophilic protein structures and antigenicity. The development of an electron microscope that allows the observation of samples in a non-vacuum environment started from the demand for direct observation of samples in liquid or gas at high resolution by EM.

\subsection{Environmental SEM}

In 1979, Danilatos et al. developed the environmental scanning electron microscope (ESEM), which allowed the observation of samples in chambers filled with air maintained at low-density by combining a differential pumping mechanism utilizing apertures for the electron beam path and a low-vacuum secondary electron detector. ${ }^{[2]}$ Since the observation took place in thin air at about $1 / 100^{\text {th }}$ of atmospheric pressure, moisture evaporated from the sample during observation. Thus, the sample had to be imaged before it dried (Fig. 2 left). To resolve this issue, the sample was generally kept at low temperature, about $5^{\circ} \mathrm{C}$, to reduce the evaporation speed of water.

\subsection{The environmental capsule and its limits}

The environmental capsule was born from the desire to realize a stable aqueous environment under high atmospheric pressure. It has a window that is transparent to an electron beam, and was developed for TEM and scanning electron microscopy (SEM). In fact, the first capsule was developed immediately after the invention of EM. ${ }^{[3]}$ Carbon and collodion films were used for the early capsule windows. However, these environmental capsules did not become very popular due to the weakness of the films. When the film broke because of the uneven flatness of an area, the sample tended to contaminate the EM column. Recently, these films were replaced by strong silicon nitride ( $\mathrm{SiN}$ ) films or other films that were originally developed for the microfabrication of semiconductors, ${ }^{[4]}$ and the environmental capsule evolved dramatically due to this increased stability. ${ }^{[5]}$ However, the capsule interior is basically a closed system or a quasi-closed system with a volume of a few tens of microliters or less (Fig. 2 left). Sensitive primary cell culture directly from organs and the culture of nerve cells with high oxygen demand can be problematic, and neither long-term culture nor the addition of reagents from the outside are easy. Since the environmental capsule used for SEM only has one window, on the upper side, it is simpler than the capsule used for TEM, which has windows on both the upper and lower sides. However, cells and tissues tend to sink to the bottom due to gravity. Moreover, attaching tissue cross-sections to the film, labeling with antibodies in immuno-EM, and washing are not easy within this small container.

\section{Development of ASEM, an inverted SEM}

To overcome the above issues, we developed the ASEM (Fig. 1) ${ }^{[6]}$ A strong $100 \mathrm{~nm}$ thick $\mathrm{SiN}$ film window $(0.25$ $\mathrm{mm} \times 0.25 \mathrm{~mm}$ ) was inserted into the bottom of a dish, and samples on the film were observed from below using an inverted scanning EM. Since the nomenclature ASEM has appeared several times in the history of EM development, we add some explanation. Initially, Danilatos referred to the
A

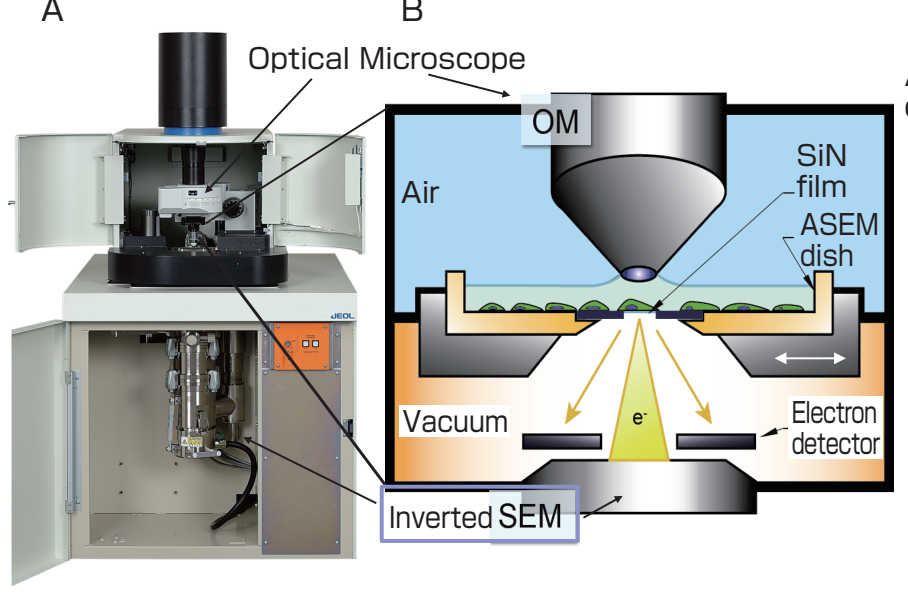

C

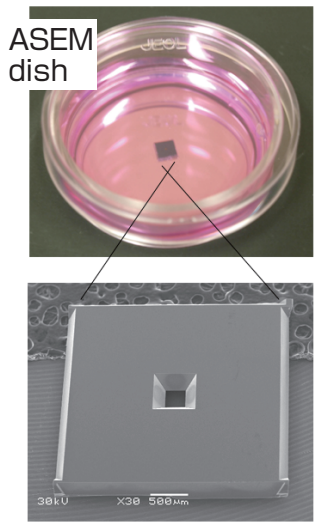

Fig. 1 Principle of ASEM

(A) The OM is installed opposite to inverted SEM, and the ASEM dish with electron-transparent film in the bottom is set between the two microscopes.

(B) The ASEM dish can be removed and used for cell culture in an incubator. The approximately 700 atom-thick SiN film (100 nm thick) of the dish was born from the semiconductor manufacturing process and is sufficiently strong to support a vacuum. The electron beam penetrates the film and irradiates the cells in the solution. The reflected electrons pass back through the film and are detected by the discoid BEI detector.

(C) ASEM dish. A square silicon chip is embedded in the center of the bottom. The lower image is the enlarged image of the chip as seen from the bottom of the dish. The center of the dish is etched to expose the SiN film window. The images are modified and reproduced from [11]. 
environmental (low vacuum) SEM as an atmospheric SEM $(\mathrm{ASEM}),{ }^{[2]}$ but the name of this device was later changed to ESEM. Next, Green and Kino ${ }^{[7]}$ called the microscope in which a film is attached to the underside of a regular SEM column, where the electron beam is released, an ASEM, and Ackerley et al ${ }^{[5]}$ called the method that uses a regular SEM and an environmental capsule to separate sample and vacuum, ASEM. Our microscope is characterized by an inverted SEM that allows the observation of samples in a container that is open to the atmosphere, rather than within a capsule. Therefore, we decided to use the name ASEM for the fourth time because we believe it is an appropriate description for our atmospheric pressure EM and hope that people will use this name. Our ASEM is totally different from the Danilatos' ESEM, but closer to the environmental capsule in the point that a film is used to separate the sample from the microscope vacuum. Samples are observed in air or in water, at atmospheric pressure.

The $35 \mathrm{~mm}$-diameter, removable ASEM dish, which is the sample holder we devised for our ASEM, enabled cell culture within a $\mathrm{CO}_{2}$ incubator to mimic the gas configuration and humidity in our body. Moreover, due to this dish format, OM techniques that had already been developed, such as antibody-labeling and washing of cultured cells, could be utilized. Since in-solution observation was now possible, the time required to prepare samples became almost the same as for OM. The actual observation procedures are as follows. The cells are inoculated onto the ASEM dish and cultured in the incubator. After culturing for several hours to several days, the dish is placed onto the ASEM sample stage using the O-ring, and the interior of the inverted SEM column beneath the dish is vacuum pumped for about one minute.
First, the behavior of the cultured cells is observed using the OM installed above the sample; this allows a wide field of view to be scanned at low magnification. Chemical fixation is done at the moment when desired cellular changes occur in the target cells, and the cells are immersed in $10 \mathrm{mg} / \mathrm{ml}$ glucose solution and observed at high magnification using the electron beam scanned through the SiN film from below (Figs. 1A, B). Since the component proteins are identified by labeling them with different fluorescent colors to analyze their distribution using the above OM, FluoroNanogold ${ }^{[8]}$ or quantum dots $^{[9]}$ that have fluorescence and electron dense body can be used to label the antigens for correlative opticalelectron observation.

\subsection{Components of the ASEM}

Figure 2 shows the scenario of ASEM development. The technological elements that comprise the ASEM are the electron-transparent film, the dish, the SEM and the OM. SiN film was adopted because of its strength. We optimized the semiconductor manufacturing process, and with the cooperation of Dr. Yoshiyuki Watabe of the Yamagata Research Institute of Technology and others, we succeeded in fabricating a SiN film window with a thickness of only 15 $\mathrm{nm}$ (about 100 atoms of $\mathrm{Si}$ or N). A CVD process and wet etching were employed to boost the fabrication precision, and we checked that the film would not break under pressure differences of up to 2 atmospheres. ${ }^{[6]}$ The OM and SEM were designed to be positioned above and below this window (Fig. 2). The film has an open structure and was embedded in the bottom of a dish so that nerve cells with high oxygen demand could be cultured. It was important that the dish could be removed for cell culture in an incubator, and that high-throughput observation could be achieved by the use of

\section{Development scenario for ASEM}

Problems of conventional technology

\begin{tabular}{|c|}
\hline $\begin{array}{c}\text { Environmental SEM (ESEM) } \\
\text { Development of differential } \\
\text { pumping }\end{array}$ \\
\hline \\
\hline $\begin{array}{c}\text { Development of gaseous } \\
\text { electron sensor }\end{array}$ \\
Disadvantage: sample is \\
under low vacuum (1/100 \\
atmospheric pressure) \\
\hline Environmental capsule \\
$\begin{array}{c}\text { Use conventional films; } \\
\text { carbon collodion }\end{array}$ \\
$\downarrow$ \\
Development of strong film \\
Disadvantage: Quasi-closed system \\
with volume of a few-ten $\mu$ or less
\end{tabular}

Three components

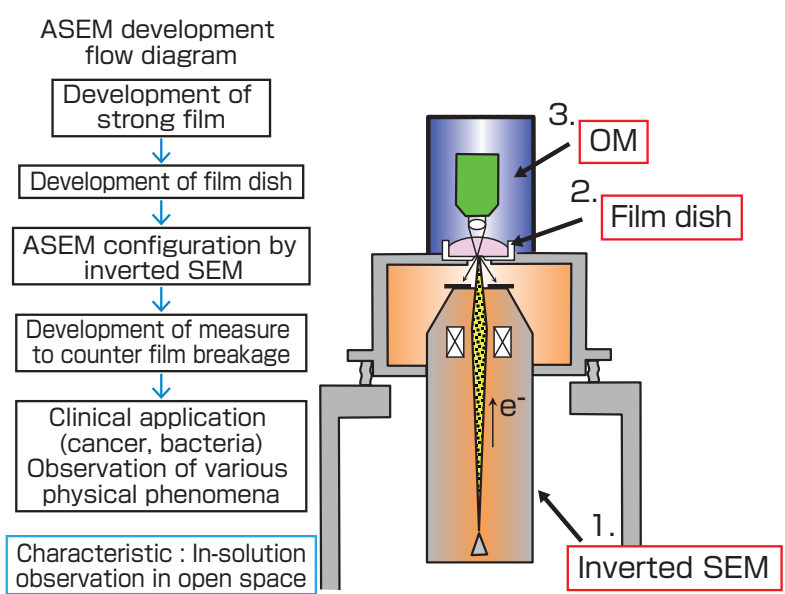

Fig. 2 Scenario for the development of ASEM

The goal is to observe samples in solution in an open space using SEM, and to enable application to clinical diagnosis and the study of physical phenomena. The basic development of the device involved the following: (1) to develop an inverted SEM; (2) to develop a disposable dish-shaped sample holder with a film window sealed above the inverted SEM; and (3) to enable simultaneous observation by OM and SEM by placing an OM above the dish. This figure was modified and reproduced from [6]. 
disposable plastic materials. ${ }^{[10]}$

\subsection{Development of the ASEM (film) dish: Fabrication of the electron beam transparent film, its durability, and resolution}

Part of the electron beam is scattered by the film. Therefore, the qualities of the film, particularly its thickness and evenness, are given as the factors that determine the resolution of the ASEM. As can be expected from the theory of electron beam scattering, the resolution gradually increased as the film becomes thinner. This tendency is clear at low acceleration voltages, where the penetration ability of the electron beam is low. However, in a commercial device, a $100 \mathrm{~nm}$ thickness is employed to reduce the possibility of film breakage and maintain a resolution of $8 \mathrm{~nm}$. Moreover, using semiconductor manufacturing technology, we succeeded in developing an eight-window chip, and developed a dish with high observation efficiency by incorporating this chip into the device.

\subsection{Mechanism of the ASEM}

The SiN film seals the end of the inverted SEM column, separating it from the atmosphere and allowing the microscope column to be kept under vacuum. ${ }^{[6]}$ The film is embedded in the bottom of the dish at the center, and the samples are either cultured directly on it prior to observation, or placed on it at the time of observation. The dish is doubly sealed to the stage by an O-ring at the side and a discoid rubber sheet at the bottom, and the evacuation is completed within about one minute. The electron beam irradiates the sample by penetrating the film from below, and part of the beam bounces back as backscattered electrons. The discshaped backscattered electron detector is placed at the exit of the SEM column. The backscattered electrons pass through the film, are quantified at each scan position by this detector, and are converted to images (Fig. 1B). The OM is installed above the sample, and same-field simultaneous observation is carried out using both microscopes. The main feature is that objects immersed in a large amount of solution $(2 \mathrm{ml})$ can be observed. A defense system is present in case the SiN film breaks. Although the SiN film is strong, it can be accidentally pierced by sharp chips or other things.

\subsection{Measures against film breakage}

If the film breaks, a three-step defense mechanisms come into play as described below. ${ }^{[10]}$ When the sensor beneath the film senses loss of vacuum, the shutter below immediately closes and the container above the shutter captures the liquid. At the same time, the air-leak valve functions, and stops further flow through the pinhole by setting the underside of the film to atmospheric pressure. Moreover, the liquid that passes before the shutter closes stops at the fine orifice through which the electron beam passes, located in the middle of the inner pipe of the column, preventing contamination of the electron gun. Because of these mechanisms, the ASEM can resume operation as soon as some parts have been exchanged. The interior of the column does not have to be cleaned. ${ }^{[10]}$

\section{Application of the ASEM to cell biology}

The cell cultured on the ASEM dish can be manipulated from the outside while it is being monitored by OM, and can be observed by SEM after fixation and staining. The resolution of the ASEM is $8 \mathrm{~nm}$. The following experiments were primarily conducted to observe the organelles, cytoskeletons and molecular complexes of cells. ${ }^{[1]]}$ Immuno-labeling was achieved and applied to primary cultures of neurons; primary neuron culture is known to be difficult in a conventional environmental capsule.

\subsection{ASEM observation of the cytoskeletons}

The cytoskeleton determines the shape of the cell, acts as a scaffold for molecular localization, and provides rails to transport substances within the cell. The microtubules are formed by tubulin polymerization, and play important roles in cell division, flagellar movement, and the formation of neural circuits. Figure 3 shows ASEM images of microtubules of renal fibroblast cell line COS7 that were treated by chemical fixation, permeabilization using $\operatorname{Triton}^{\mathrm{TM}} \mathrm{X}-100$, primary labeling with anti- $\alpha$-tubulin antibody, ${ }^{[1]}$ secondary labeling with Alexa Fluor ${ }^{\circledR} 488$ FluorNanogold ${ }^{\mathrm{TM}}$-Fab' antibody ${ }^{[8]}$ and gold enhancement. ${ }^{[12]}$ This series of procedures can be accomplished simply by changing the solution in the ASEM sample dish, and the task is completed within two or three hours. In the ASEM, the microtubules are mainly visualized
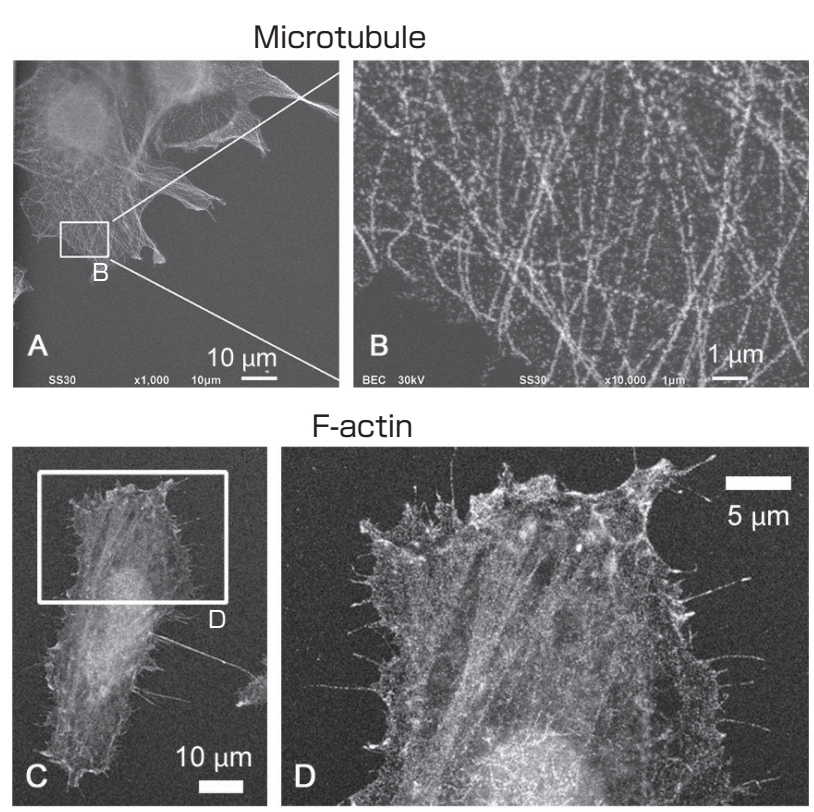

Fig. 3 Immuno-ASEM of microtubules and F-actin

(A) In-solution immuno-EM image where the $\alpha$-tubulin of COS7 cell is labeled with fluorescence and gold (FluoroNanogold), and goldenhanced. (B) At high magnification, each strand of the microtubule can be observed. (C) F-actin of Hela cell was labeled with phalloidinFluoroNanogold, and gold-enhanced. (D) Enlarged image. Observation in low background without autofluorescence. The images were modified and reproduced from [11]. 
as white lines running from the center to the periphery of the cells (Fig. 3A). When enlarged, these lines are seen to consist of a series of gold particles about $20 \mathrm{~nm}$ in diameter (Fig. $3 \mathrm{~B})$. Note that the background is extremely low.

Of course, labeling for ASEM is not restricted to immunoEM. Actin filaments (F-actin) are known to play an important role in the cell movement as well as in synapse formation and plasticity. Figures $3 \mathrm{C}$ and D show the distribution of F-actin in the cytoplasm and at the cell periphery. In this case, the F-actin of Hela cells was labeled with mushroom phalloidingold, and gold enhanced as in Fig. 3A. The actin bundles are thought to be mostly stress fibers.

\subsection{Up to what thickness can the ASEM see?}

When ASEM observation is conducted at an acceleration voltage of $30 \mathrm{kV}$, what depth from the film can be observed? As in Fig. 3, microtubules were labeled and comparative observations were made with a confocal fluorescence microscope. As seen in Fig. 4 A and B, the protrusion (arrow head) and the round structure (arrow) in the ASEM image were not present in the confocal image of the bottom part of the film (uppermost image). Rather, these structures were only observed when the sections sampled were at a distance from the film (the lower images of A). Together with other data, ${ }^{[13]}$ this shows that a depth of $2-3 \mu \mathrm{m}$ is observable by the ASEM. ${ }^{[11]}$ The latter value decreased to about $1 \mu \mathrm{m}$ as the accelerating voltage decreased to $10 \mathrm{kV} \cdot{ }^{[13]}$ Since synapses and neurites of cultured neurons are generally within this range, they are thought to be sufficiently observable by ASEM.

\subsection{Observable depth predicted by electron pathway simulations}

The trajectory of each electron was simulated according to scattering probabilities calculated by the Monte Carlo method. As the acceleration voltage increases from $20 \mathrm{kV}$ to $30 \mathrm{kV}$, the irradiation depth of the electron beam increases (Fig. 5). Therefore, the observable depth is also expected to increase. The results agreed well with Fig. 4. In fact, the predicted observable depth at $10 \mathrm{kV}$ is $1 \mu \mathrm{m}$, but deepens to $2 \sim 3 \mu \mathrm{m}$ at $30 \mathrm{kV} .^{[13]}$

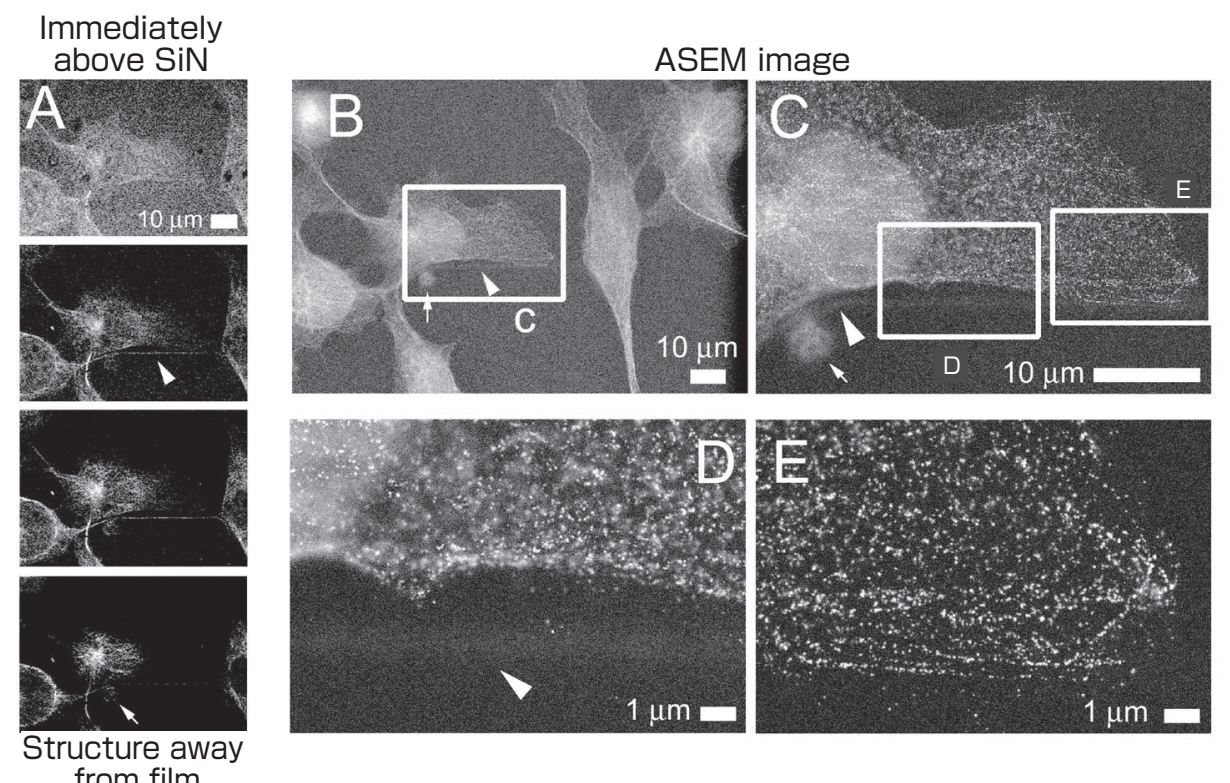

\section{Fig. 4 Depth observable by ASEM}

As in Fig. 3A, the $\alpha$-tubulins of the COS7 cells were labeled with FluoroNanogold, and the depth observable by ASEM was measured by comparison with confocal fluorescence microscopy. (A) Confocal fluorescence microscopy image. Top image, the SiN film surface; bottom image, $1.32 \mu \mathrm{m}$ above the film surface; central images, intermediate positions. (B-E) Corresponding ASEM images. It was estimated that 2-3 $\mu \mathrm{m}$ is the depth observable by ASEM. ${ }^{[11][13]}$ The images were modified and reproduced from [11].

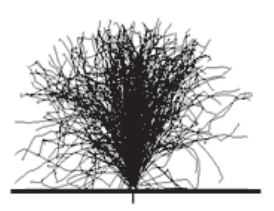

$20 \mathrm{kV}$

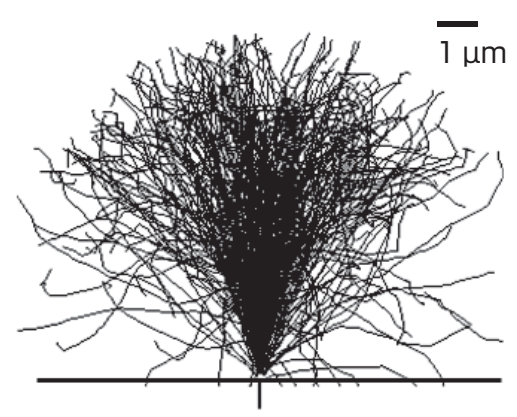

$30 \mathrm{kV}$
Fig. 5 Simulation of the electron pathways

The trajectories of electrons in bulk carbon were calculated by Monte Carlo simulations to understand electron imaging by ASEM. The simulations were made for acceleration voltages of $20 \mathrm{kV}$ and $30 \mathrm{kV}$. The calculation supports the results shown in Fig. 4. The images were modified and reproduced from [6]. 


\subsection{Reconstruction of the cytoskeleton during synapse formation in primary cultured nerve cells}

The synapse is the basic unit of the neural network. Its size is small generally being $50-500 \mathrm{~nm}$, and many of the axons and dendrites that comprise the network are minute. For their observation, the resolution of the EM is more appropriate than that of the OM. However, to count the synaptic connection of cultured cells by TEM, thin sectioning is normally required. This difficult job delivers thin horizontal slices cut against the culture plane after the neurons have been embedded in resin. On the other hand, ASEM allows observation of synapses without embedding or slicing, and we were even able to observe a small $50 \mathrm{~nm}$ spine.

Figures 6A-D show the observation of the growing axon terminal (growth cone) of a neuron. Primary cultures of the mouse hippocampal neurons (pyramidal neurons) were grown on an ASEM dish coated with poly-L-resin for four days, ${ }^{[1]]}$ and labeled for F-actin after fixation and cell membrane permeation. Then, the growth cone shown in the white frame of Fig. 6A was observed using the ASEM. Fine
F-actin can be seen, developed like bicycle spokes in the lamellipodia of the axon terminal (Fig. 6C and D). Homer $1 \mathrm{c}$ coexists in the center of the spoke structure (Fig. 6B, yellow green). This agrees with the idea that Homer receives the $\mathrm{Ca}^{2+}$ signal information and intervenes with the action of the growth cone by controlling the actin polymerization. When the synapses were formed after 14 days of culture, the synaptic sites (Figs. 6G, H) could be identified using the Homer 1c (Fig. 6F, green) localized in the spine as landmarks. Microtubules existed as the backbone of the dendrites, and were hardly present in the synaptic sites (Fig. 6G). Also, it was newly discovered that microtubules inside the dendrite run diagonally (in a spiral) (Figs. 6I, J). It is known that the synapses are distributed spirally on dendrites to maximize space usage, and this could be the structural basis.

\subsection{Dynamic rearrangement of signal transmitting molecules; visualization of the $\mathrm{Ca}^{2+}$ perception mechanism of the CRAC ion channel}

The $\mathrm{Ca}^{2+}$ sensor STIM1, which is a membrane protein of the endoplasmic reticulum (ER), is the sensor of the plasma
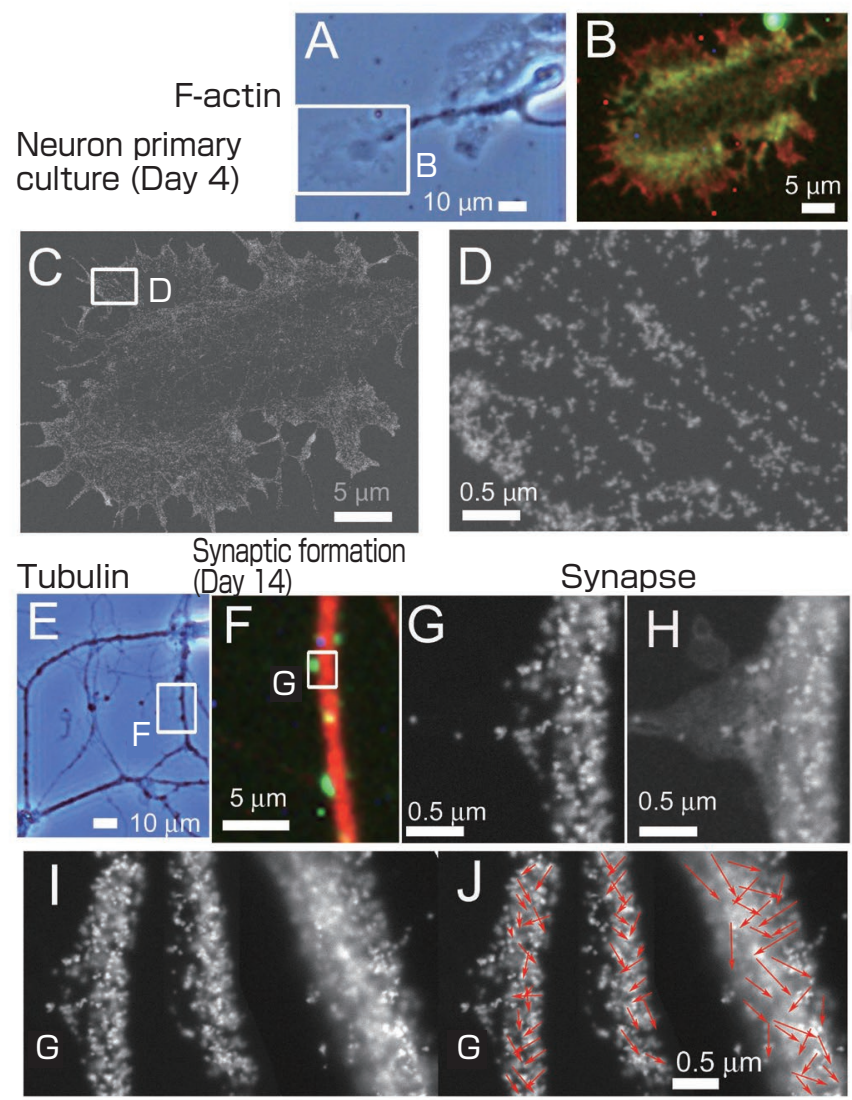

Spiral structure of microtubule bundles

Fig. 6 Growth cone and synapse formation in primary culture nerve cells

Primary cultures of Homer 1c-EGFP transgenic mouse hippocampal pyramidal cells were grown on an ASEM dish coated with poly-L-lysine. (A) Phase contrast OM image of the growth cone four days later. The F-actin present was labeled with red and gold using FluoroNanogold. (B) Fluorescence images and (C, D) ASEM images after gold-enhancement. The F-actin was observed in a bicycle spoke-like form on the lamellipodia of the growth cone. (E) Phase contrast image 14 days after culture. Synapses had formed. (F) Fluorescent image. (G-I) ASEM images. (G)Tubulin of the synapse and $(\mathrm{H})$ further stained with heavy metal. (I, J) The microtubules in the dendrites ran diagonally (in a spiral) against the long axis. Microscopy through a whole synapse and growth cone became possible at high resolution. The images were modified and reproduced from [11]. 
membrane CRAC ion channel. STIM1 monitors the $\mathrm{Ca}^{2+}$ concentration within the ER, and is thought to open the CRAC channel when it perceives the lack of $\mathrm{Ca}^{2+}$. Figure 7 shows the observed distribution of STIM1 on the ER membrane before and after depleting $\mathrm{Ca}^{2+}$ in independent experiments. ${ }^{[11]}$ In a steady state when the ER was storing $\mathrm{Ca}^{2+}$, a correlative observation conducted by fluorescence labeling of the ER marker PDI (Fig. 7A) showed that the gold particles indicating STIM1 were distributed throughout the ER (Figs. 7B, C). Once $\mathrm{Ca}^{2+}$ was depleted, the STIM1 molecules gathered near the cell membrane in spots (Figs. 7E-G). The molecules gathered and joined together onedimensionally making winding lines; the ASEM visualized this for the first time (Fig. 7G). Since the STIM1 molecule is asymmetric, the patterning suggests that the molecules bond head-to-tail. This STIM1 polymer bonds further with Orai of the cell membrane to form an active ion channel supercomplex. The positional changes of the CD44 glycan receptor on the plasma membrane, were also successfully imaged. This receptor is important in early development and

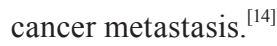

\section{Clinical application}

\subsection{Possibile use of the ASEM as a clinical diagnosis device}

The use of the ASEM in clinics and hospitals as a diagnostic tool is expected, since long sample pre-treatment is not necessary and the direct in-solution observation of samples is possible. To visualize the characteristics that serve as the indices for diagnosis, we have developed heavy metal staining solutions for the following two cases, and have started to develop the diagnostic method. Further, to widen the observation area, we have also developed an eightwindow sample dish.

\subsubsection{Identification of infectious bacteria}

Infection by bacteria is one of the most serious factors that threaten life. Since treatment differs according to the type of bacteria, a way of identifying the species that does not require time-consuming steps, such as culture, is strongly in demand. Mycoplasma, which is recently gaining attention as a cause of pneumonia and other diseases, has only about $1 / 25^{\text {th }}$ of the cell volume of $E$. coli. In the recent epidemic, over $90 \%$ of the bacteria were antibiotic-resistant, and quick diagnosis is particularly in demand. However, due to the small size of Mycoplasma, diagnosis is difficult at all stages of disease, including early diagnosis. Mycoplasma mobile found in fish gills was observed with the ASEM as a model. After fixation and following membrane permeabilization, it was stained with heavy metal solutions consisting mainly of uranium and lead. The visualized bacteria had a cap structure at the more elongated end of the cell, nucleic acid in the round posterior end, and a varied structure in the middle (Fig. 8A). ${ }^{[15]}$ These structures are characteristic, and are likely to become extremely prominent markers for diagnosis. When the "leg" gliding machinery that supports transport was labeled with gold-tagged antigens and gold-enhanced as in Fig. 3A, a waistband-like distribution was seen on the cell surface (Fig. 8B bottom), which indicated that the immunolabel may be useful for diagnosis. We also succeeded in the detailed observation of bacillus. ${ }^{[16]}$ These results show that the ASEM may potentially speed up the diagnosis of infectious diseases.

\subsubsection{Identification of metastasis; application to rapid intraoperative cancer diagnosis}

During cancer resection surgery, intraoperative cancer diagnosis is sometimes required. The most important index for rapid cancer diagnosis is the size of the nucleus. Generally, tissues are frozen, cut into about $3 \mu \mathrm{m}$-thick

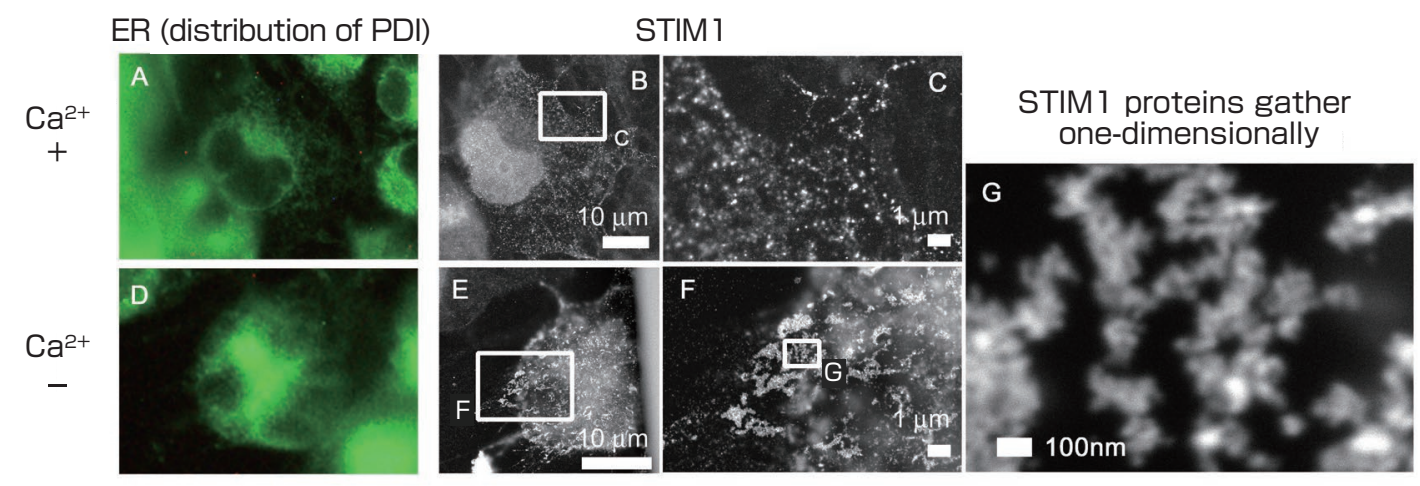

Fig. 7 Gathering of STIM1 proteins due to $\mathrm{Ca}^{2+}$ decrease in the endoplasmic reticulum (ER)

The STIM1 subunits that are the $\mathrm{Ca}^{2+}$ sensors of CRAC channels are distributed on the cell ER (A-C), and aggregate in spots at the puncta near the plasma membrane when they sense $\mathrm{Ca}^{2+}$ depletion (D-G). The colored panels are fluorescent images and the black-white panels are ASEM images of gold-labeled STIM1, and enlargements of the areas in white boxes are shown to the right. In the image with the maximum enlargement, the gold particles labeling STIM1 are joined together linearly, and we thus propose that the asymmetrical STIM1 molecules bond one-dimensionally (G). STIM1 is thought to form an ion channel supercomplex with Orail between the ER and the plasma membrane. (A, D) The ER marker PDI was fluorescent labeled. The distribution of the ER is shown. Here, we present the results of the COS7 over expression system, but similar string-like molecular gathering was observed for the intrinsic STIM1 in T cells, ${ }^{[11]}$ and this phenomenon is considered to occur universally. The images were modified and reproduced from [11]. 
slices, stained with hematoxylin eosin, and observed using an OM. The extent of resection is sometimes determined from the nucleus size; cancer cells usually have bigger nuclei. However, the required cryo-sectioning is not easy, taking at least 15-30 min for all procedures per a sample. Since time is pressing during an operation, only a limited number of regions can be examined, which is also a problem. First, we developed, by trial and error, a staining method for the nucleus that can be used with ASEM. We found that the nucleus could be stained at high contrast when the cultured cell was fixed, first with $4 \%$ paraformaldehyde (PFA) and then with $1 \%$ glutaraldehyde (GA), and stained, first with 10 -fold diluted Ti -Blue (platinum-Blue) solution and then with $2 \%$ phosphotungstic acid (PTA) solution. ${ }^{[17]}$ When the stained tissue was placed on the SiN film of an ASEM dish and imaged, nuclei $2-3 \mu \mathrm{m}$ above the surface could be observed. We fixed and sectioned a normal lung and a breast- cancer metastasized lung from mice. The tissues were crosssectioned into thick slabs, stained, and observed using the ASEM. The nuclei stood out as white structures, and the large nuclei of cancer cells were clearly discernable (Fig. 9). ${ }^{[17]}$ Since this method does not require cryo-sectioning, it has the potential to dramatically speed up and simplify the intra-operative diagnosis. Observing as many areas as possible with the microscope is expected to increase the diagnostic accuracy.

\section{Application to energy and material fields}

\subsection{Electrochemical reaction; application to battery development}

Observing the electrochemical reaction in electrolytic solution in situ and at high resolution is important for the development of batteries and electrolytic solutions. Therefore,
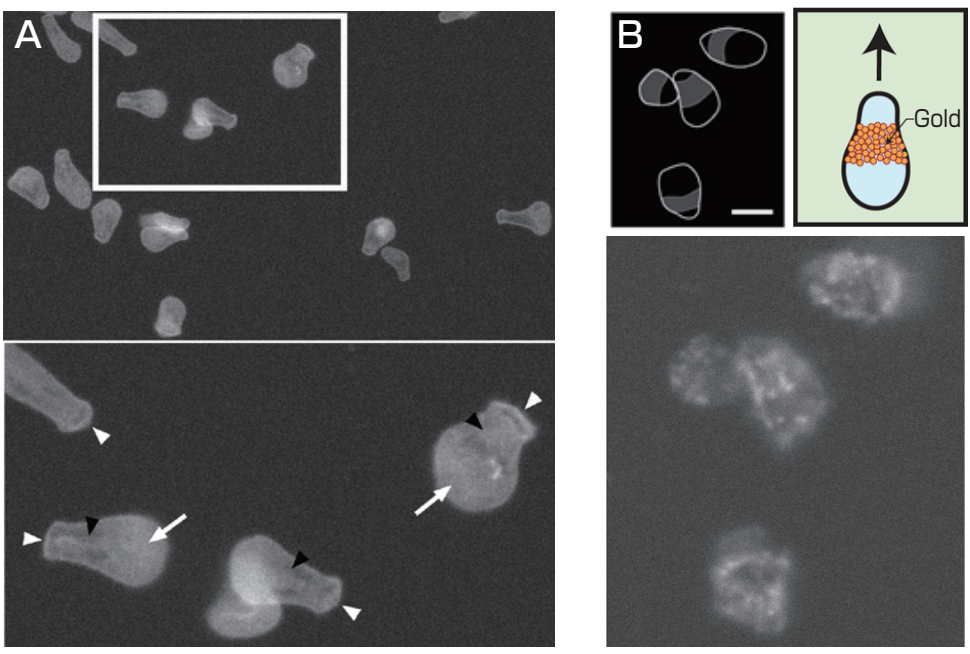

Fig. 8 ASEM images of Mycoplasma mobile

(A) Staining was done with heavy metal. The lower image is an enlargement. (B) Immuno-EM image of the foot protein complex Gli349. The cells were counter-stained with heavy metal after gold labeling. The upper image is a schematic diagram, and the cells move in the direction of the arrow. Micoplasma generally has $1 / 25^{\text {th }}$ of the volume of $E$. coli. The fine interior structures of this cell were observed in solution for the first time using the ASEM. The images were modified and reproduced from [15].
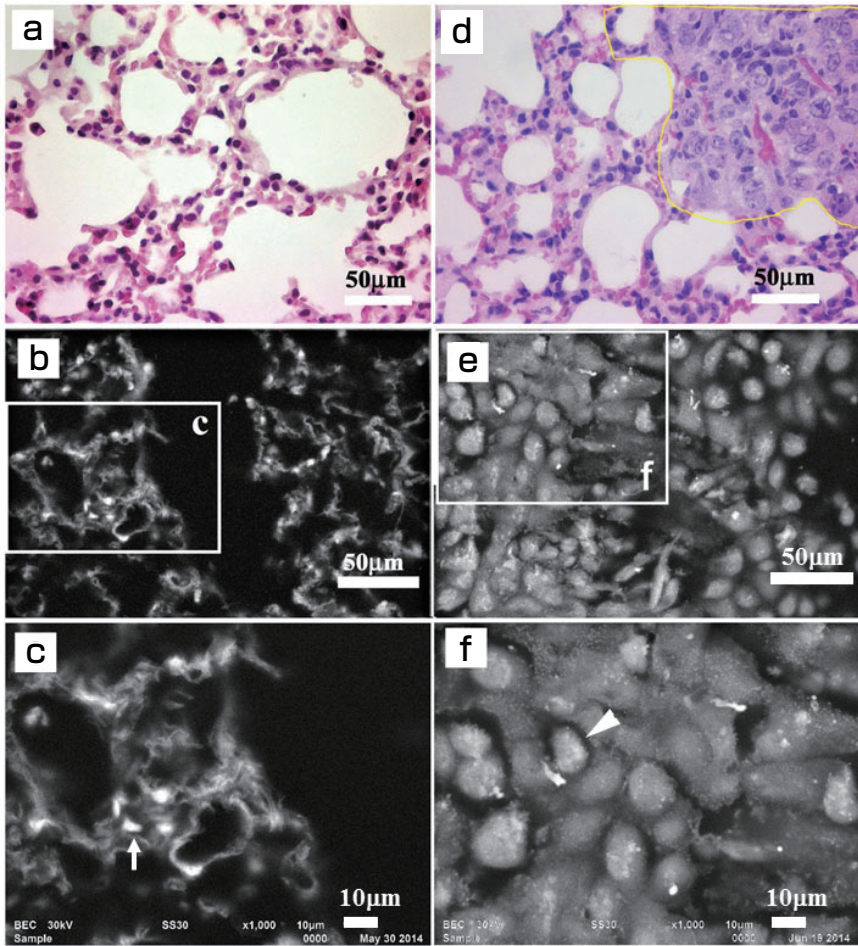

Fig. 9 Applicability of ASEM to cell nucleus-based cancer diagnosis of tissues

Comparative observation of normal lung and lung from mice metastasized by breast cancer. Nuclei near the tissue surface could be clearly observed in solution just by staining the tissue with TiBlue and phosphotungstic acid (PTA): cell cytoplasm was also weakly stained. (a) OM of an hematoxylin/eosin-stained thin-section of normal lung. Nuclei and the cytoplasm are stained blue and red, respectively. (b-c) ASEM image of independently prepared normal lung tissue slab stained with Ti-Blue and PTA. Alveoli with alveolar ducts, a vein system and trachea are visible. Normal size nuclei are observed (arrow). The tissue was placed on the SiN film and observed using ASEM. (d-f) Comparative observation of lung metastasized by breast cancer cells. Regular alveolar systems are only faintly discernable, but most of the space is occupied by cells of different shapes with larger nuclei (arrowhead), i.e., cancer cells. Because the nuclei near the surface of the tissue block could be observed, this method could be applied to intra-operative cancer diagnosis, which mainly depends on the size of nuclei. The images were modified and reproduced from [17]. 
we developed an electrochemical ASEM dish equipped with two electrodes set $100 \mu \mathrm{m}$ apart on the SiN film (Figs. 10A, B). ${ }^{[18]}$ The electrodes were made by sequentially depositing $10 \mathrm{~nm}$ thick titanium and $100 \mathrm{~nm}$ thick gold onto the SiN film by the sputtering method, and then processing the layers by photolithography and wet etching.

The area around the cathode was continuously observed in a saturated $\mathrm{NaCl}$ solution by ASEM under the following conditions: 5,000x magnification, $0.15 \mathrm{sec}$ per frame, and four times integration. Immediately after monitoring, voltage was applied between the anode and cathode. The ASEM images recorded $3 \mathrm{sec}$ (Fig. 10C) and $6 \mathrm{sec}$ (Fig. 10D) after voltage application are shown. The growth of the treelike structure from the cathode to the anode was recorded on video in real time. The sample was dehydrated after observation and the tree-like precipitates were analyzed by SEM energy dispersive spectrometry (SEM-EDS), and were found to be gold.

When the ASEM is used, the deposition of metal by electrochemical reaction in aqueous electrolyte can be observed in real time. The real time observation of electrochemical phenomena in electrolytic solutions in the vacuum of a conventional SEM is limited to solutions, such as some ionic liquids, that have a low vapor pressure. This is thought to be the first SEM observation made using a commonly employed aqueous electrolyte that is difficult to maintain in a vacuum.

\subsection{Application to micro-wiring, melting and solidification of solder}
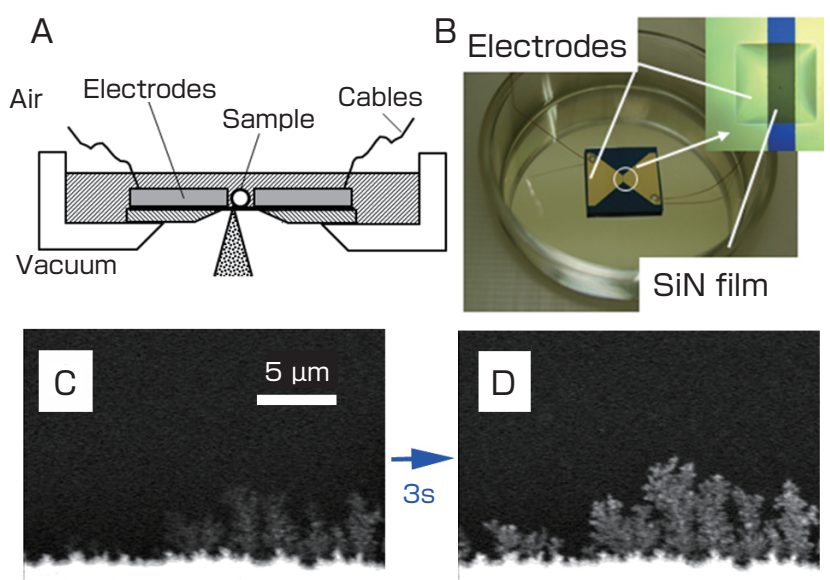

Fig. 10 Electrochemical ASEM dish (A, B) and the observation of an electrochemical reaction in the electrolytic solution (C, D)

Two electrodes were formed at a $100 \mu \mathrm{m}$ interval on the SiN film (A, B). After applying $2.1 \mathrm{~V}$, ASEM was used to observe the area around the cathode; after $3 \mathrm{sec}(\mathrm{C})$ and $6 \mathrm{sec}$ later (D). The dendritic precipitate grew from the cathode in the direction of the anode. The observation of phenomena occurring in aqueous electrolyte was shown to be possible. The images were modified and reproduced from [18].
To observe phenomenon that change depending on the temperature in solution and gas, we developed a temperaturecontrollable ASEM dish. ${ }^{[18]}$ This has a similar structure to the standard dish, but includes a heater and a thermometer to change and control the temperature, and the body is made of titanium to withstand temperature rise (Fig. 11A). The SiN film could withstand the high temperature at which a type of solder ( $\mathrm{Sn}: 42 \mathrm{wt} \%$; Bi: $58 \mathrm{wt} \%$ ) melted. At the temperatures shown in Fig. 11B, the solder goes from the solid to the molten state and vice versa, with accompanying morphological changes (Figs. 11C-D). ${ }^{[18]}$ At a temperature of $145{ }^{\circ} \mathrm{C}$, the solder melted and the contrast of the ASEM image became uniform, and when it was cooled slowly to $130{ }^{\circ} \mathrm{C}$, the metals segregated (Fig. 11C). When the temperature was increased again to $150{ }^{\circ} \mathrm{C}$ and then rapidly decreased to 115 ${ }^{\circ} \mathrm{C}$, the morphology of the segregation changed (Fig. 11D). The segregation varied depending on the cooling conditions. In electric circuit formation using solder, minute quantities of volatile ingredients such as pine resin are important, and the ASEM is expected to contribute to research and development in this field.

\section{Future issues}

The quick in-solution observation possible using the ASEM allows the realization of experiments under various conditions. Further, and of great importance, in-solution correlative observation by OM and EM can be accomplished by one device.

Since observation of samples open to the air and the use of

A

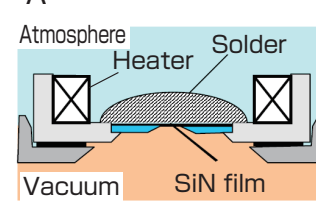

$\mathrm{B}$
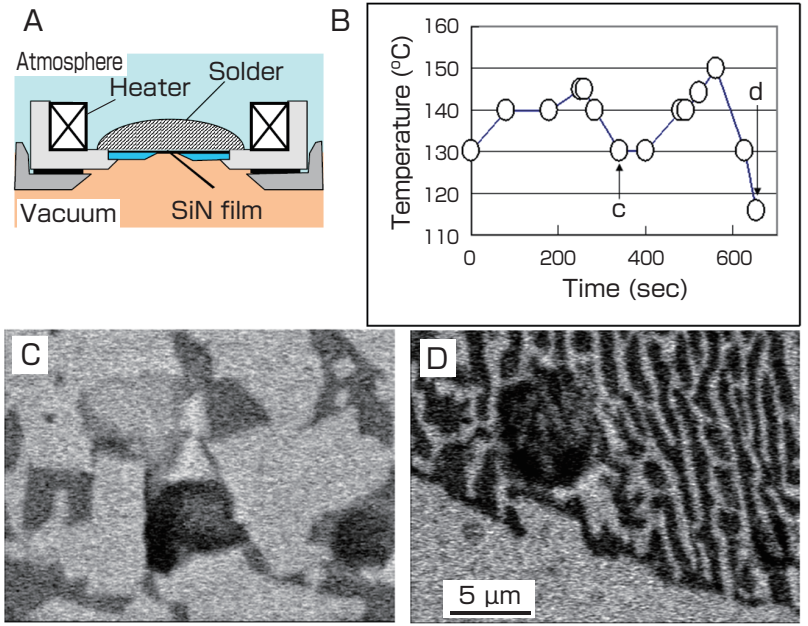

Fig. 11 Temperature changeable ASEM dish (A), temperature change (B), and ASEM images of solder (C, D)

The dish was equipped with a heater and a thermometer (A). The molten solder solidified as the temperature decreased $(C, D)$. The bright-dark contrast stood out due to segregation, but the form differed according to the cooling conditions. Observation became possible even when volatile ingredients such as pine resin were present. The images were modified and reproduced from [18]. 
two microscopes was made possible by the development of the ASEM dish, it has become possible to observe various dynamic phenomena including the electrochemical reactions where gas is produced. For example, in an electrochemical reaction the bubbles produced float upward, so it is difficult to image them with the inverted SEM, but they can be observed by OM. At the same time, since observation is made from below using the inverted SEM, it is less likely to be affected by the bubbles, even in a phenomenon where gas is produced. Moreover, this microscope brings two new advantages to cell research. One is that various cells that were difficult to culture on an electron-transparent film in a capsule can now be cultured ${ }^{[19]}$ and the other is that the efficiency of labeling and washing is increased. In the ASEM dish, cells can be cultured in a $\mathrm{CO}_{2}$ incubator in a relatively large culture volume $(2 \mathrm{ml})$, compared to the small volume of the environmental capsule. Therefore, the effect of evaporation can be minimized, the ingredients of the culture solution can be maintained at a certain level, and the oxygen and $\mathrm{CO}_{2}$ concentrations are stable due to gas exchange across a large liquid surface. Cell adhesion to the SiN film increases if the film is treated by a surface coating agent developed primarily for glass. ${ }^{[1]]}$ This is probably because the surface of the SiN film becomes SiOx during the manufacturing process, being oxidized by the fabrication process, and takes on a glass-like property. The ease of labeling and washing using the ASEM dish allows the immuno-EM method to have almost the same high-throughput as the OM. As a result, multiple-sample analysis and screening become simple, and there were cases where 40 dishes per day were observed. Since the preparation of the sample is conducted entirely in solution, the preservation of antigenicity is extremely good. We tested about 100 antibodies for cell labeling, and ASEM observation was possible with all of them. The majority of the antibodies used were mouse monoclonal antibodies, and it is expected that many commercially available antibodies can be employed. By adding the process of staining by heavy metal, it is possible to accentuate certain cell structures around the antigen. Uranyl acetate and PTA mainly accentuate protein and nucleic acid, while osmium tetroxide accentuates oil droplets and membrane structures. ${ }^{[6]}$ By changing the acceleration voltage of the ASEM, it is possible to estimate the distance of the target to the SiN film. While this is similar to confocal $\mathrm{OM}$ in function, the principal is different.

High-throughput ASEM can observe diverse samples in a short time at high resolution. Compared to other EMs, the operation and sample preparation is extremely easy to learn. One factor is that, since the SiN film is set at the same height at all times, the focus position stays in the same place. As well as the results described here, the ASEM can observe fine protein crystals in a crystallizing solution without staining. ${ }^{[20]}$ The application of ASEM to clinical diagnosis is one of our goals. It is expected that intra-operative diagnosis for cancer can be sped up by measuring the nucleus size with the ASEM.
However, it is necessary to develop automated recognition and coloring software using image processing, since it is essential to distinguish between the nuclei and the cytoplasm, as in the standard OM-based diagnosis. In addition, ASEM is expected to contribute to the rapid diagnosis of infectious bacteria. ${ }^{[15]-[17]}$ To accomplish this, observation protocols, including staining methods for general diagnosis mainly for pneumococcus, bacillus, MRSA, and viruses, are strongly in demand. The ASEM can be applied to drug discovery, food chemistry, polymer chemistry, cement, and other fields. Moreover, it can be applied widely to various fields of physics and physical-property research, such as materials science ${ }^{[18]}$ and nanoscience.

\section{Acknowledgement}

We thank Makoto Tsuyuki, Takeshi Sato, Yoshio Ishimori, Mitsuru Koizumi, and Yasuji Ogawa of JEOL Ltd. for the manufacture of the ASEM. We thank Dr. Yoshiyuki Watabe of the Yamagata Research Institute of Technology and Yuji Konyuba of JEOL Ltd. for the manufacture of the SiN film. We thank Drs. Yuusuke Maruyama, Tatsuhiko Ebihara, Nassirhadjy Memtily, Sachie Abe and Tomoko Okada of AIST for their researches using ASEM. Part of this research was conducted with the support of matching funds from AIST and JEOL Ltd., Grant-in-Aid for Scientific Research for Priority Area "Bio-supermolecule", and Grant-in-Aid for Scientific Research for Innovative Area "Structural Cell Biology", KAKENHI by METI and the Strategic Basic Research Program CREST.

\section{References}

[1] M. Nagasawa, Y. Nakagawa, S. Tanaka and I. Kojima: Chemotactic peptide fMetLeuPhe induces translocation of the TRPV2 channel in macrophages, J. Cell. Physiol., 210 (3), 692-702 (2007).

[2] G. D. Danilatos: An atmospheric scanning electron microscope (ASEM), Scanning, 3 (3), 215-217 (1980).

[3] I. M. Abrams and J. W. McBain: A closed cell for electron microscopy, J. Appl. Phys., 15 (8), 607-609 (1944).

[4] T. Tomie, H. Shimizu, T. Majima, M. Yamada, T. Kanayama, H. Kondo, M. Yano and M. Ono: Three-dimensional readout of flash X-ray images of living sperm in water by atomicforce microscopy, Science, 252 (5006), 691-693 (1991).

[5] C. Ackerley, C. Nielsen and C. Hawkins: Experiences with wet capsule imaging exploring the potential for live cell imaging, Microsc. Microanal., 12 (Supp. S02), 428-429 (2006).

[6] H. Nishiyama, M. Suga, T. Ogura, Y. Maruyama, M. Koizumi, K. Mio, S. Kitamura and C. Sato: Atmospheric scanning electron microscope observes cells and tissues in open medium through silicon nitride film, J. Struct. Biol., 169 (3), 438-449 (2010).

[7] E. D. Green and G. S. Kino: Atmospheric scanning electron microscopy using silicon nitride thin film windows, $J$. Vac. Sci. Technol. B, 9 (3), 1557-1558 (1991).

[8] R. D. Powell, C. M. Halsey and J. F. Hainfeld: Combined fluorescent and gold immunoprobes: reagents and methods 
for correlative light and electron microscopy, Microsc. Res. Techniq., 42 (1), 2-12 (1998).

[9] B. N. Giepmans, T. J. Deerinck, B. L. Smarr, Y. Z. Jones and M. H. Ellisman: Correlated light and electron microscopic imaging of multiple endogenous proteins using Quantum dots, Nat. Methods, 2 (10), 743-749 (2005).

[10] H. Nishiyama, M. Koizumi, K. Ogawa, S. Kitamura, Y. Konyuba, Y. Watanabe, N. Ohbayashi, M. Fukuda, M. Suga and C. Sato: Atmospheric scanning electron microscope system with an open sample chamber: configuration and applications, Ultramicroscopy, 147, 86-97 (2014).

[11] Y. Maruyama, T. Ebihara, H. Nishiyama, M. Suga and C. Sato: Immuno EM-OM correlative microscopy in solution by atmospheric scanning electron microscopy (ASEM), $J$. Struct. Biol., 180 (2), 259-270 (2012).

[12] R. D. Powell and J. F. Hainfeld: Silver- and gold-based autometallography of Nanogold, Gold and Silver Staining: Techniques in Molecular Morphology (G. W. Hacker, J. Gu, (eds.)), CRC Press, Boca Raton, FL, 29-46 (2002).

[13] M. Suga, H. Nishiyama, T. Ebihara, T. Ogura and C. Sato: Atmospheric electron microscope: limits of observable depth, Microsc. Microanal., 15 (Supp S2), 924-925 (2009).

[14] T. Murai, Y. Maruyama, K. Mio, H. Nishiyama, M. Suga and C. Sato: Low cholesterol triggers membrane microdomaindependent CD44 shedding and suppresses tumor cell migration, J. Biol. Chem., 286 (3), 1999-2007 (2011).

[15] C. Sato, S. Manaka, D. Nakane, H. Nishiyama, M. Suga, T. Nishizaka, M. Miyata and Y. Maruyama: Rapid imaging of mycoplasma in solution using atmospheric scanning electron microscopy (ASEM), Biochem. Biophys. Res. Commun., 417 (4), 1213-1218 (2012).

[16] T. Kinoshita, Y. Mori, K. Hirano, S. Sugimoto, K. Okuda, S. Matsumoto, T. Namiki, T. Ebihara, M. Kawata, H. Nishiyama, M. Sato, M. Suga, K. Higashiyama, K. Sonomoto, Y. Mizunoe, S. Nishihara and C. Sato: Immunoelectron microscopy of primary cell cultures from genetically modified animals in liquid by atmospheric scanning electron microscopy (ASEM), Microsc. Microanal., 20 (2), 469-483 (2014).

[17] N.Memtily, T.Okada, T.Ebihara, M.Sato, A.Kurabayashi, M.Furihata, M.Suga, H.Nishiyama, K.Mio And C.Sato: Observation of tissues in open aqueous solution by atmospheric scanning electron microscopy: applicability to intraoperative cancer diagnosis, Int J Oncol, 46, 18721882(2015)

[18] M. Suga, H. Nishiyama, Y. Konyuba, S. Iwamatsu, Y. Watanabe, C. Yoshiura, T. Ueda and C. Sato: The atmospheric scanning electron microscope with open sample space observes dynamic phenomena in liquid or gas, Ultramicroscopy, 111 (12), 1650-1658 (2011).

[19] K. Hirano, T. Kinoshita, T. Uemura, H. Motohashi, Y. Watanabe, T. Ebihara, H. Nishiyama, M.Sato, M. Suga, Y. Maruyama, N. M. Tsuji, M. Yamamoto, S. Nishihara and C. Sato: Electron microscopy of primary cell cultures in solution and correlative optical microscopy using ASEM, Ultramicroscopy, 143, 52-66 (2014).

[20] Y. Maruyama, T. Ebihara, H. Nishiyama, Y. Konyuba, M. Senda, T. Numaga-Tomita, T. Senda, M. Suga and C. Sato: Direct observation of protein microcrystals in crystallization buffer by atmospheric scanning electron microscopy, Int. $J$. Mol. Sci., 13 (8), 10553-10567 (2012).

\section{Authors}

Toshihiko OGURA

Completed the doctorate course at the Department of Systems and Information Engineering, Graduate School of Engineering, Toyohashi University of Technology in 1997. Researcher, Omron Institute of Life Science Co., Ltd. in 1997; Research Fellow, Electrotechnical Laboratory (currently AIST), Agency of Industrial Science and Technology in

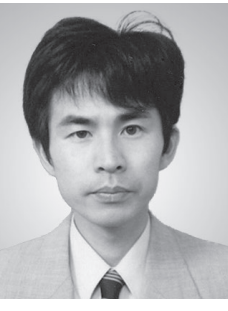
2000; Researcher, Neuroscience Research Institute, AIST in 2003; and currently, Senior Researcher, Structure Physiology Research Group, Biomedical Research Institute, AIST. In this paper, was in charge of the planning, development, and configuration of the device.

\section{Hidetoshi NishiYAma}

Completed the master's course at the Department of Applied Physics, Tokyo Institute of Technology in 1992. Joined the Central Research Laboratory, Hitachi, Ltd. in 1992; joined JEOL Ltd. in 2005; and became Deputy General Manager, SEM Development Section, JEOL Ltd. in 2014. In this paper, was in charge of the planning and development of the device,

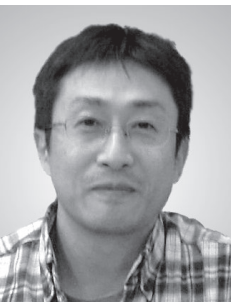
as well as the software development for bio and materials use.

\section{Mitsuo Suga}

Completed the master's course at the Department of Physics, Graduate School of Science, Tohoku University in 1989. Joined the Central Research Laboratory, Hitachi, Ltd. in 1989; joined JEOL Ltd. in 2000; and became Deputy Director, Advanced and Fundamental Technology Center, JEOL Ltd. in 2013. Obtained Doctor of Engineering at Chiba University in 2014. In this paper, was in charge of the planning and development of the device, as well as the software development for materials use.

\section{Chikara SATo}

Completed the doctorate at the Graduate School of Science, Tohoku University in 1989. Doctor of Science. Joined the Electrotechnical Laboratory (currently AIST), Agency of Industrial Science and Technology in 1989; joined AIST in 2001; and currently, Group Leader, Structure Physiology Research Group, Biomedical Research Institute, AIST. In this paper,

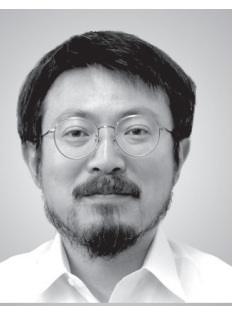
was in charge of the planning and development of the device, as well as the software development for bio use. 


\section{Discussions with Reviewers}

\section{Overall evaluation}

Comment (Shingo Ichimura, Nagoya University and Hideto Taya, J-Space Inc.)

This paper discusses the construction of a scenario, the selection of elemental technologies, and the progress on the atmospheric scanning electron microscope (ASEM) developed by utilizing the ultrathin film technology for semiconductors, and its use in insolution observation that is highly in demand in the bio field. I think this paper offers value for Synthesiology.

While the sample had to be kept in a vacuum in the conventional EM method, with the development of the in-solution observation by ASEM as described in this paper, the rapid and high resolution observation of the activities inside cells became possible for the bio fields. In the future, this method is expected to be applied to clinical medicine, as well as materials science and nanoscience fields.

\section{Overall structure \\ Comment (Shingo Ichimura)}

When I do a search on Google Scholar, the term and concept of atmospheric scanning electron microscope (ASEM) can be traced back to the 1980s. I think you should completely change the way this paper is written based on whether the main point of this paper is: (1) the development of ASEM, (2) the application of ASEM, or (3) both.

If it is (1), what is different from the conventional ASEM? What kind of new elemental technologies did you incorporate into the existing technology to compose the system (device)? What was the background that led you to this concept? If you describe these, I think the characteristics of the paper that deal with a synthesiological approach will become more apparent. Since you already have the description of the basic characteristic, please reorganize the paper while explaining the historical course of ASEM development, and clarify the characteristic of your development.

If it is (2), rather than listing the examples of observation, the paper will become a paper related to synthesiological approach if you explain the following: what were known using which method up to this moment (conversely, what were the limitations); what became known through the application of the ASEM method (in addition, what did you consider as the technological element to overcome the limitations of the conventional method); and how clear did your understanding of the essence of the observed subjects become.

\section{Answer (Chikara Sato)}

The main point of this paper is (3). Following your comment, I reorganized the paper to combine (1) and (2) as follows.

First, I totally reorganized and rewrote Chapters 1 3 considering the history of EM development. First, I added the development history of in-solution observation EM in Subchapters 2.1 2.3, and explained the limitations of the conventional method in Subchapter 2.1 "Conventional electron microscopy and its limit," Subchapter 2.2 "Environmental SEM," and Subchapter 2.3 "Progress of environmental capsule and its limit." I also added the origin of the name ASEM and the changes that occurred in the first paragraph of Chapter 3. For elemental technologies, I newly added Fig. 2 "Scenario for the development of ASEM" and some explanations in Subchapter 3.1 "Components of the ASEM." The developmental element based on how much the understanding of the sample progressed was also added.

\section{Scenario}

Comment (Shingo Ichimura)

The historical flow of the ASEM is now fairly clear. However,
I think it is lacking in the point of "summarizing the outline of the scenario, selection of elements, and relationship and integration of the elements into one "diagram of the scenario." Please add a single diagram that explains the elements (characteristics) and problems of the development methods, for which you have described the history (environmental SEM and environmental capsule), and present how the selection and deletion as well as the addition of new functions have led to this new development.

In accompaniment to the diagram, I think you should further explain how the characteristics "inversion of the SEM column" and "use of SiN film" were selected as elements that would be newly added.

\section{Comment (Hideto Taya)}

The scenario diagram shows the elements of the ASEM, and is insufficient as the scenario that should provide the framework of this paper. If the title of this paper is "Development of an insolution observation method," you must show the course and process of research that led to the establishment of the in-solution method, and I think the development of ASEM was one of the steps. Please show the elemental technologies that were needed to attain the goal of the paper "establishment of the in-solution observation method," along with the issues that had to be solved. Answer (Chikara Sato)

As you indicated, I reorganized Fig. 2. While showing the problems of the present technologies, I presented the flow of R\&D to realize the "in-solution observation in open space."

\section{Relationship between the title and "Introduction" Comment (Shingo Ichimura)}

The current title is "Development of an in-solution observation method." This title gives the reader expectations for contents about the clarification of reactions and phenomena in solution, as you show later in the examples of non-bio fields. On the other hand, the "Introduction" is clearly limited to the bio field, and does not state the significance or necessity of the "in-solution observation method." Therefore, it is necessary to reconsider whether to keep the title and change the "Introduction" (point out the significance and necessity of "in-solution observation method"), or keep the "Introduction" and change the title.

You do not need the chapter on "Discussion." This is a paper for a journal characterized by the discussion of synthetic approach throughout the paper.

Answer (Chikara Sato)

I worked on the beginning part of the "Introduction" and revised the whole paper.

\section{Description that emphasizes the "application of ASEM" Comment (Shingo Ichimura)}

There are two non-bio field examples that you provide: one for electrochemical reaction and the other for micro-wiring. I don't think the latter, "melting and solidification of solder" is appropriate as an application example because the title is "Development of an in-solution observation method" (I think soldering is an in-gas reaction). In accordance to the title, please consider using (or deleting) this example or adding other examples.

\section{Answer (Chikara Sato)}

In circuit formation by solder, the minute volatile ingredients such as pine resin are important, and micro-wiring is a field where future development can be expected. Therefore, I used these examples as the possibilities of application. Upon reflecting on your indication, I added the expression, "In electric circuit formation using solder, minute quantities of volatile ingredients such as pine resin are important, and the ASEM is expected to contribute to research and development in this field." 